\section{Cahiers de Narratologie}

Analyse et théorie narratives

$10.1 \mid 2001$

La voix narrative

\title{
L'écho et l'écart : la voix narrative dans Clarissa (1747-48) de Samuel Richardson
}

\section{Hélène Dachez}

\section{(2) OpenEdition \\ 1 Journals}

\section{Electronic version}

URL: http://journals.openedition.org/narratologie/6961

DOI: 10.4000/narratologie.6961

ISSN: 1765-307X

\section{Publisher}

LIRCES

\section{Printed version}

Date of publication: 1 January 2001

Number of pages: $335-345$

ISBN: 2914561032

ISSN: 0993-8516

\section{Electronic reference}

Hélène Dachez, "L'écho et l'écart : la voix narrative dans Clarissa (1747-48) de Samuel Richardson", Cahiers de Narratologie [Online], 10.1 | 2001, Online since 28 October 2014, connection on 23 February 2021. URL: http://journals.openedition.org/narratologie/6961 ; DOI: https://doi.org/10.4000/ narratologie.6961 


\title{
L'ECHO ET L'ÉCART : LA VOIX NARRATIVE DANS CLARISSA (1747-48) DE SAMUEL RICHARDSON
}

\author{
Hélène DACHEZ
}

Université Toulouse 2 - Le Mirail

«Ce ne sont pas les ressemblances mais les différences qui se ressemblent "

Claude Lévi-Strauss, Le Totémisme aujourd'hui (1962)

Même si Samuel Richardson était moins instruit que son rival Henry Fielding, sa connaissance de la mythologie grecque ne fait aucun doute. Dès 1739, l'auteur, qui n'est encore qu'imprimeur, propose au public une version des Fables d'Esope, inspirée de celle de Robert l'Estrange'. La voix de Richardson, non créatrice ici, n'est que l'écho de celle de son prédécesseur. En revanche, en composant Clarissa en 1747-48 et en faisant allusion de façon directe et indirecte à l'histoire d'Echo (et de Narcisse), il montre comment l'écho (au sens de répétition) sous-tend la voix narrative et devient, de manière originale, un principe créateur ${ }^{2}$.

A deux reprises, Richardson attire l'attention du lecteur (extradiégétique) sur la correspondance entre la mythologie et ses personnages. Lovelace, devant le refus de sa future victime, envisage de commettre " a rape worthy of Jupiter!» (165). Il fait référence à l'une des variantes du mythe, selon laquelle la nymphe Echo retenait l'attention de Junon par ses bavardages et permettait au dieu de se livrer à des conquêtes

1 On pourra consulter sur ce point les pages 233-34 et 297 de l'ouvrage suivant: John Carroll, ed., Selected Letters of Samuel Richardson (Oxford: Clarendon, 1964).

${ }^{2}$ Samuel RICHARDSON, Clarissa ; Or, the History of a Young Lady, ed. Angus Ross (1747-48; Harmondsworth: Penguin, 1985). 
amoureuses illicites sans être dérangé par son épouse. Par la suite, c'est à Ovide, auteur de cette version, que se compare le libertin. "Ovid was not a greater master of metamorphoses than thy friend » (412), claironne-t-il, en expliquant à Belford qu'il a fait passer Clarissa, qu'il vient d'enlever, pour sa sœur.

Plus souvent, c'est en filigrane que le mythe est intégré au roman ${ }^{3}$. Punie par Junon pour sa trahison, Echo est contrainte à ne jamais parler la première et à répéter les derniers mots prononcés par autrui ${ }^{4}$. Cette situation correspond à celle de Clarissa. Sa famille censure sa voix propre (que le lecteur entend dans les lettres à Anna Howe) et lui faire réciter un discours qu'elle lui dicte. Son père la prive de l'initiative de la parole : « No protestations, girl ! » lui enjointil, « No words - I will not be prated to ! - I will be obeyed ! I have no child - I will have no child » (64). M. Harlowe, dont les propos reposent sur l'écho, exige que la voix de sa fille ne soit que la répétition de la sienne, sans écart aucun.

Cet ordre est exaucé par la suite lorsque, trop affectée pour commenter la malédiction de son père, l'héroïne dit : «I enclose the letter itself. Transcribe it I cannot. ... I can only repeat » (508). C'est d'ailleurs à partir de cet écho épistolaire que la santé de Clarissa se détériore, comme celle d'Echo, dont Ovide décrit le sort : " les soucis qui la tiennent éveillée épuisent son corps misérable, la maigreur dessèche sa peau.... Il ne lui reste plus que la voix et les os ; sa voix est intacte, ses os ont pris, dit-on, la forme d'un rocher » $(82)^{5}$. Comme celui de l'Oréade, le corps de Clarissa s'étiole peu à peu. Elle envie les saints, car "they have shaken off the encumbrance of body » (974) et souhaite faire de même. Son enveloppe

${ }^{3}$ Françoise GRAZIANI (Dictionnaire des mythes grecs, éd. Pierre Brunel [Paris : Rocher, 1988]) résume le mythe narré par Ovide, au Livre III des Métamorphoses et par Longus dans Daphnis et Chloé (527-32).

4 Voir OVIDE, Les Métamorphoses, trad. Georges Lafaye (Paris : Belles Lettres, 1972) 81.

${ }^{5} \mathrm{Si}$ elle est affectée par l'anathème paternel, la jeune fille l'est aussi par l'échec de son amour avec Lovelace, comme Echo l'est du sien pour Narcisse, ainsi qu'en témoigne le diagnostic du Dr. H. : «a love case ... There is one person in the world who can do her more service than all the faculty » (1081). 
charnelle s'amenuise et Belford la compare à «a lovely skeleton » (1231).

D'Echo, il ne reste plus que la voix, qui résonne dans une caverne, " the cave where Echo lies. " ${ }^{6}$ Clarissa, de même, désire se réfugier dans une grotte ("some obscure corner" [651]) et son corps se pétrifie. "There is a sort of stone... [that] will become as hard as marble, and then with difficulty it yields to the chisel. So this lady " (844), remarque Lovelace $^{7}$. La remarque de l'héroïne : "I am but a cipher » (567), intéressante à plus d'un titre, synthétise le rapprochement des voix d'Echo et de Clarissa. Le SOED définit le terme comme " a nonentity », qui évoque la disparition du corps, «a ... disguised system of writing », qui reprend l'écriture allusive de Richardson, et "a continuous sounding ", son réitéré qui s'apparente à l'écho acoustique. La voix (narrative) de Clarissa retentit après sa mort, annoncée par Belford (1359). Ses paroles occupent encore les pages suivantes, sous forme des lettres à sa famille, ainsi que de son testament, lu après l'ensevelissement de son corps ${ }^{8}$.

Par ces échos narratifs, le texte semble assigner le rôle de la nymphe à Clarissa et celui de Pan (dans la version de Longus) à Lovelace. Lors de la scène de l'incendie, l'héroïne est prise de panique à l'idée qu'elle se trouve à la merci de son séducteur. Le libertin est mû par un désir sexuel jamais assouvi, tel le dieu de la fécondité. D'ailleurs, à plusieurs reprises (dans un écho structurel), il s'imagine qu'il va être père $(720,916$ et 1147$)$.

Or, un décalage (forme et signe de l'écart) intervient ici. Pour le libertin, Clarissa devient déesse de la fécondité. Il s'imagine voir " a twin Lovelace at each charming breast,

6 William SHAKESPEARE, The Tragedy of Romeo and Juliet (2.2.161).

7 Dans le tableau de Nicolas POUSSIN (Echo et Narcisse, Musée du Louvre, Paris), la nymphe qui admire Narcisse est couchée sur un rocher, position qui laisse présager que la chair va se métamorphoser en pierre.

8 Clarissa écrit à son père (L. 488, 1371), à sa mère (L. 489, 1372), à son frère (L. 490, 1373), à sa sœur (L. 491, 1374) et à ses oncles John et Antony (L. 492, 1375). 
drawing from it his first sustenance.... [She was] pressing with her fine fingers the generous flood into the purple mouths of each eager hunter by turns » (706). De même, le démembrement d'Eccho, décidé par Pan, qui ne peut se faire aimer d'elle, concerne les deux personnages. Juste avant le viol, le corps de l'héroïne est désarticulé et son discours répétitif : « in she came... her ruffles torn, and hanging in tatters... Dear Lovelace, said she, if ever - if ever - if ever -And, unable to speak another word... down she sunk, as if quite disjointed » (880-81). Il existe en effet, selon Françoise Graziani, « une analogie entre le démembrement rituel et la fragmentation des sons de l'écho" (529). Lovelace, en apprenant que sa prisonnière vient de s'enfuir de chez la Sinclair, éprouve une réaction physique semblable: "my back aching as if the vertebrae were disjointed, and falling in pieces » (741).

En s'écartant du mythe, Richardson intensifie l'écho entre ses personnages. Il montre que le libertin souffre de la même langueur que Clarissa, qui, pour sa part, devient le reflet de Narcisse (captivé par sa propre image et indifférent à tout le reste) dans sa volonté de ne plus s'occuper que d'ellemême et de se couper du monde (777). En outre, la couleur et la forme penchée du lys qui orne le cercueil de Clarissa (1306) évoquent "une fleur couleur de safran, dont le centre est entouré de blancs pétales » (Ovide, Métamorphoses 86), qui se reflète dans l'onde à l'endroit où se mirait Narcisse. L'héroïne fait inscrire sur la bière le Psaume 103, où l'homme est comparé à une fleur, et Belford la nomme " this sweet and tender blossom of a woman » (1306). En outre, comme la nymphe et Clarissa, le libertin, en apprenant le décès de cette dernière, " creeps into holes and corners » (1360) et dépérit. Alors qu'il méprisait le sommeil au début du texte (387), il se plaint ensuite d'insomnie (904). Hanté par le souvenir de Clarissa comme l'Oréade l'est par Narcisse, il est lui aussi réduit à une voix, écho qui sonne aux oreilles de Miss Howe : « his odious voice, whispering, behind [me]» (1135).

Donc, le mythe de la nymphe, avec ses variantes, résonne dans Clarissa en échos explicites et implicites, directs et inversés. Cette "coöncidence-discordance » (terme emprunté à Lucien Dällenbach) s'inscrit plus profondément 
dans le roman pour révéler le jeu entre la polyphonie et la fusion des voix, stratégie narrative fondée sur l'écho et sur l'écart ${ }^{9}$.

Le contrat d'écriture établi dans la Preface de l'ouvrage explique que Clarissa est un roman à voix multiples et réflexives, puisque les personnages proposent « reflections upon each other and each upon himself and his actions " (35). Chacun narre sa propre version des événements et il arrive que les discours se contredisent. Ce phénomène, que Gérard Genette nomme "transfocalisation », repose sur une relation spéculaire et mêle l'alternance des voix et leur confrontation $^{10}$. Par exemple, selon la famille de la victime, il ne fait pas de doute que Lovelace est responsable du décès de Miss Betterton, violée par lui et morte en couches (494). Or le personnage dédouble la voix narrative, insère l'écart dans l'écho pour démentir les accusations : "there was no rape in the case : ... he went not abroad on her account : ... she loved him and he loved her ... she owed her death to her friends' barbarity » (495). De façon semblable, le départ de Clarissa est, selon la voix entendue, soit un enlèvement, soit une fuite voulue. La jeune fille se dit entraînée par son ravisseur contre son gré (380). La voix du libertin, écho fidèle de celle de Clarissa, confirme les dires de cette dernière : " [she flew] from the friends she was resolved not to abandon to the man she was resolved not to go off with » (400). Toutefois, le témoignage du serviteur qui a assisté à la scène infirme les deux précédents. Il s'agit d'une fuite volontaire et non d'un enlèvement : " he saw you run with [Lovelace] as fast as you could set foot to ground " (406). Si l'écho s'accompagne d'écart, la réciproque est vraie, car les termes du valet « run... as fast as you " reprennent un à un (si l'on excepte le changement des pronoms personnels) les mots de Clarissa : «I ran as fast as he » (380). Comme le note Thomas O. Beebee, « Clarissa is not a story, but a collection of stories, ... [it]

${ }^{9}$ Lucien DÄLLENBACH, Le Récit spéculaire : essai sur la mise en abyme (Paris : Seuil, 1977) 49.

${ }^{10}$ Gérard GENETTE, Nouveau discours du récit (Paris : Seuil, 1983) 45. 
exists only in its variations. ${ }^{11}$ La voix narrative se métamorphose au fil des lettres, phénomène dû au genre épistolaire car, selon Regina Bocheneck-Franczakowa, «il y a ... autant de savoirs du [narrateur] qu'il y a de lettres, la connaissance qu'il possède à l'instant donné pouvant être mise en cause à l'instant qui suit. »12

En fait, ici, l'un des paradoxes de la polyphonie narrative est que les voix des personnages se fondent et se confondent. Anna Howe donne la clé de cette osmose vocalique en expliquant comment Clarissa fait bénéficier ses voisines de son expérience: "she dropped in the needful instruction, and left the instructed unable to decide whether the thought ... came primarily from herself, or from the sweet instructress » (1468). Lovelace aussi laisse parler Belford dans une lettre où il imagine les remarques et les critiques de son compagnon. Les deux voix se rejoignent en un pseudodialogue : "And what! (methinks thou askest with surprise)» (426), « And what could the lady say to this ? methinks thou askest » (425).

Or il n'est pas rare que deux voix, en fusionnant, se dissocient de leur discours originel. M. Harlowe, qui ne réprouvait pas une union entre Lovelace et sa fille cadette, finit par faire siennes les objections de son fils et s'oppose au mariage. La voix du fils contamine celle du père au point de la métamorphoser. Au fur et à mesure que le roman se forme, l'écart se creuse entre les lettres que Lovelace rédige, en même temps que son discours se rapproche de celui de Clarissa. Selon Lucien Dällenbach, le texte "se contrelit : alimenté par les contradictions qu'il génère, il se multiplie par scissiparité toujours relancée et va-et-vient réflexif » (188). Le parfait libertin renie ainsi ses pratiques licencieuses (1359), et la voix de Clarissa, qui s'exprime en "grave soliloquies " (141), s'insinue peu à peu en lui qui, après le viol, regrette de

11 Thomas O. BEEBEE, Clarissa on the Continent: Translation and Seduction (University Park : Pennsylvania State UP, 1990) 26, 42.

12 Regina BOCHENECK-FRANCZAKOWA, Le Roman épistolaire à voix multiples en France de 1761 à 1782 : problèmes de forme; destinateur-destinataire (Krakow: Nakladen Uniwersytetu Jagiellonskiego, 1986) 32. 
ne pouvoir retrouver la gaieté qui le caractérisait : «I cannot help writing in a more serious vein than usual » (887). Si, lors de l'outrage, le corps de l'héroïne est envahi contre son gré, la voix du libertin, dans un écho inversé, semble être absorbée par celle de la jeune fille, qui devient alors sa conscience. Dans un processus proche de la ventriloquie, les paroles de Clarissa se font entendre par la bouche (i.e., dans les lettres) du libertin, qui reconnaît le phénomène : "she has a friend here (clapping my hand on my breast) that pleads for her with ... irresistible eloquence » (837). Le texte insiste encore sur l'osmose des voix dans la lettre où Lovelace se livre au meurtre symbolique de sa conscience (et de Clarissa, unies dans l'emploi du pronom personnel «she »): "there she lies, weltering in her blood! Her death's wound have I given her ! - But she was a thief, an impostor, as well as a tormentor. She had stolen my pen ... and thus she wrote with it, in a hand exactly like my own » (848). Le personnage renforce la fusion en accusant sa victime d'une action (le vol et la fabrication de fausses lettres) à laquelle lui-même se livre, puisqu'il subtilise des messages de Miss Howe pour en extraire un alphabet qui lui permet d'écrire à Clarissa en se faisant passer pour sa confidente (754). Une fois l'héroïne morte, sa voix continue de retentir en Lovelace : " my pen will slide into this gloomy subject, whether I will or not ... All I took pen to write for is however unwritten " (1429). L'écart est grand entre les flambées de triomphe joyeux et confiant du début du texte et le ton assombri, empreint de regrets de la suite.

Le lecteur comprend alors que la création romanesque est sous-tendue par le jeu entre l'écho et l'écart. La répétition à l'identique, en ce sens, ne saurait être que stérile et facteur (ou signe) de blocage. Par elle, le texte s'enlise, comme le note René Passeron : « elle ... ne débouche sur rien. C'est celle de Sisyphe, ... une répétitivité sans issue. ${ }^{13}$ Chez Richardson, la voix de l'éditeur s'élève pour signaler que la répétition verbatim est supprimée du corps du texte, par souci de dynamisme (411). L'écho n'a de valeur et de portée narrative que s'il est associé à l'écart. Clarissa le sait, qui se désole de

13 René PASSERON, "Poiétique et répétition", Création et répétition, éd. René Passeron (Paris : Clancier-Guénaud, 1981) 11. 
ne pas pouvoir envoyer à son amie des nouvelles. « What can I write », demande-t-elle, " that I have not already written ? » (173). De même, pour Lovelace, privé de Clarissa, la répétition de jours identiques n'a aucun sens, et il préfère y mettre un terme : " my mind tires and sickens with impatience on ruminating upon scenes that can afford [no] ... variety » (1476). Gilles Deleuze établit la distinction entre deux types de répétition et c'est sur la deuxième que Clarissa est composé :

l'une de ces répétitions est du même, ... l'autre est du Différent, et comprend la différence. L'une a des termes et des places fixes, l'autre comprend essentiellement le déplacement et le déguisement. ... L'une est statique, l'autre dynamique. L'une est une répétition nue, ... l'autre est une répétition vêtue, dont les masques, les déplacements et les déguisements sont les premiers, les derniers et les seuls éléments ${ }^{14}$.

C'est donc par le jeu entre les correspondances et les divergences que la voix narrative, dans Clarissa, se développe et progresse - principalement selon trois procédés récurrents : l'accrétion, l'antithèse (c'est-à-dire l'écho inversé) et la variation sémantique et phonique.

Selon Françoise Graziani, la parole de la nymphe est " révélatrice des vérités cachées sous des apparences trompeuses, ... créatrice de sens, ... porteus ... de connaissance " (531-32). Cet effet se conjugue à la fonction relevée par Pierre Fontanier, selon qui "la Répétition consiste à employer plusieurs fois les mêmes termes ou le même tour, soit pour le simple ornement du discours, soit pour une expression plus forte et plus énergique de la passion. »15 Dans Clarissa, c'est à la répétition (accompagnée de différence) qu'est dévolu ce double rôle. L'héroïne dit son désir de poser sa plume. Poursuivre la rédaction l'obligerait à critiquer la réaction de ses proches, ce qu'elle refuse de faire. C'est par la réitération des termes identiques, mais dont le

14 Gilles DELEUZE, Différence et répétition (1968; Paris : PUF, 1993) 367-68.

15 Pierre FONTANIER, Les Figures du discours (1821; Paris : Flammarion, 1977) 329. 
sens est modifié par les mots qu'elle enserre, que sont visibles ses critiques voilées : «I will lay down my pen - But can Yet, positively, I will lay down my pen ! - (65). Comme le remarque Antoine Compagnon, « [la répétition] n'a pas de sens hors de la force qui l'agit, qui la saisit, l'exploite et l'incorpore. " ${ }^{16}$ L'écho anaphorique, qui confère à la phrase de Lovelace un rythme ternaire amplifié qui aboutit à un spondée, montre combien le motif, mêlé à l'écart, construit et fait progresser le texte : "I see », se vante-t-il, «the dear soul loves me. I see she forgives me all that's past. I see I have credit for a new score » (677). Ici, le processus se double d'une antithèse entre le passé («past») et l'avenir (« new »).

Ce procédé antithétique est fort courant dans l'esthétique de Richardson. Il concerne d'abord les structures romanesques. Trois épisodes de crise se réfléchissent en échos inversés. Clarissa est sommée par sa famille réunie au grand complet de rencontrer Solmes. Ne pouvant supporter d'être rudoyée par son frère, elle se sauve dans le jardin (308). Juste après, elle revient mais, surprise par l'ouverture subite de la porte, tombe dans une pièce vide (312). Lors du troisième épisode, ses proches emploient avec elle une douceur qui contraste avec l'intransigeance initiale, mais leurs murmures (rappels inversés des cris lors des deux premières scènes) sont le signe d'une violence contenue et prête à exploser ${ }^{17}$.

En outre, maintes phrases s'articulent sur l'antithèse. Le passif répond à l'actif et le participe passé au participe présent dans l'extrait où Clarissa dit : " one half of mankind tormenting the other, and being tormented themselves in tormenting ! » (224). Les contraires se réfléchissent dans une relation spéculaire inversée, dont les exemples sont légion. Tout finit par opposer Belford et Lovelace, " this humane and that inhuman libertine " (1194). Alors que son ami aurait pu devenir un autre Lovelace (163), c'est ce dernier qui espère l'imiter (1463). L'antithèse les rapproche et les contraste

16 Antoine COMPAGNON, «Les Mécanismes de la répétition dans le texte », Thèse, Université Paris VII, 1977, 53.

17 Clarissa note : «I heard [my uncle] say, whispering ... whispered, as audibly, M. Solmes ... said my uncle in an audible whisper " (31415). 
terme à terme : " my outside is the best of $m e$, thine the worst of thee " (1145). "The cause of your glory ... is my shame " (393), lance Clarissa à Lovelace, qui réunit et oppose l'amour et la haine : « love, which I hate, heartily hate, because 'tis my master » (148). Ici, l'adverbe " heartily », en italiques, lie les contraires et constitue le pivot de la phrase par sa position centrale et par son sens, puisqu'il concerne à la fois le cœur (l'amour) et la haine ("heartily hate »). La spécularité est encore renforcée et gauchie par la paronomase entre [heart] et [hate] ${ }^{18}$.

La voix narrative du roman participe ainsi des glissements de sens et de sons par un effet spéculaire de miroirs en cascade. L'expression « a conditional kind of liking » (135), par laquelle Clarissa définit à son amie ses sentiments pour Lovelace, subit une variation (rendue possible par le va-etvient des lettres et par le dialogue des voix), qui la modifie peu à peu. Sous la plume d'Anna, elle devient « conditional likings » (174) et est reprise par Clarissa (185), qui cherche à la justifier, mais en vain, car Anna dénonce la passion de son amie par antimétabole: " what before was only a conditional liking, I am now afraid will turn to liking unconditional » (287). L'écho inversé illustre la remarque de Bruno Duborgel, pour qui «l'ombre engendre le jumeau pour mieux le démentir et le remodeler. $\gg 19$

Aussi, semblable à la voix d'Echo dans la répétition, l'homophonie s'accompagne souvent d'un léger décalage. Lovelace joue avec les sons lorsqu'il écrit à Belford : " my predominant passion is girl, not gold; nor value I this, but as it helps me to that » (417). Le ton est plus sérieux chez Clarissa, qui explique à Anna les mouvements incessants de son séducteur : " he is forced to dodge about in hopes of a letter, and return five miles, and then to an inconvenient lodging » (241). Les allées et venues (« dodge ») s'opposent

${ }^{18}$ Pierre FONTANIER définit le procédé rhétorique, qui « réunit dans la même phrase des mots dont le son est à peu près le même, mais le sens tout à fait différent » (357).

${ }^{19}$ Bruno DUBORGEL, Préface, Figures de la répétition, éd. Bruno Duborgel (Saint-Etienne: Centre Interdisciplinaire d'Etudes et de Recherches sur l'Expression Contemporaine, 1992) 11. 
par le sens mais, à l'exception d'une lettre, s'apparentent par le son (« lodg[e]») à l'auberge, abri stable, bien qu'imparfait. Dans la phrase : " in and out of every place where I have seen the beloved of my heart, do I hurry ; in none can I tarry ; her lovely image in every one ... rushing cruelly upon me » (740), l'auteur met en relief le jeu narratif phonique grâce à la répétition verbatim («every»), à l'emploi de polyptotes («beloved»/«lovely»), ainsi qu'à l'écho antithétique (« none »/« one »). Le procédé culmine dans la paronomase entre « hurry » et « tarry ».

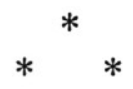

Ainsi, dans Clarissa, l'écho et l'écart, séparés et joints, différents et identiques, sont en tension constante. Le rapport dialectique qui les unit donne vie et dynamisme à la voix narrative. Le roman de Richardson, tissu de voix multiples qui se réfléchissent, se répondent, s'affirment et s'infirment, s'élabore et progresse par et dans la répétition, grâce aux nuances, aux dissonnances et aux décalages que le romancier intègre au procédé itératif. L'esthétique de l'écrivain, où se fondent le même et l'autre, la circularité et la linéarité, se fait l'écho (microcosmique) du profond changement (macrocosmique) par lequel, dès le XVII ${ }^{\mathrm{e}}$ siècle, la société et la littérature se mettent à rejeter le mimétisme pur et servile, que résume Michel Foucault dans l'expression « les quatre similitudes », au profit d'une création qui cependant ne renie pas tout principe de filiation ${ }^{20}$.

20 Ces «quatre similitudes" sont «la convenientia»(33), «l'aemulatio » (34), "l'analogie » (36) et le «jeu des sympathies" (38). Voir Michel FOUCAULT, Les Mots et les choses: une archéologie des sciences humaines (Paris : Gallimard, 1966). 